\title{
Determination of the increased risk of developing atrial fibrillation in fibromyalgia syndrome
}

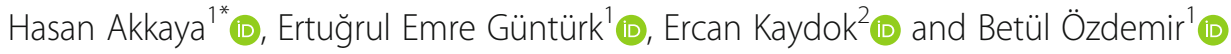

\begin{abstract}
Introduction: Atrial fibrillation (AF) is the leading cause of ischemic stroke and is one of the most common arrhythmias. Previous studies have shown that impaired diastolic functions, $\mathrm{P}$ wave dispersion (Pd), and prolonged atrial conduction times (ACT) are associated with increased incidence of atrial fibrillation (AF). The aim of this study was to evaluate diastolic functions, $\mathrm{Pd}$, and $\mathrm{ACT}$ in fibromyalgia syndrome (FMS) patients to determine whether there is an increase in the risk of developing AF.
\end{abstract}

Methods: The study included a total of 140 female patients (70 FMS group, 70 healthy control group). Pd was evaluated using 12 lead electrocardiography (ECG), and diastolic functions and ACT with echocardiography. The ECG and echocardiographic evaluations were performed by different cardiologists blinded to the clinical information of the subjects.

Results: There was no difference between the two groups in laboratory and clinical parameters. Patients with FMS had significantly higher echocardiographic parameters of ACT known as left-sided intra-atrial (13.9 \pm 5.9 vs. $8.1 \pm 1.8, p<0.001)$, right-sided intra-atrial $(21.9 \pm 8.2$ vs. $10.4 \pm 3.5, p<0.001)$ and interatrial [40 (25-64) ms vs. 23 (14-27) ms $p<0.001$ ] electromechanical interval (EMI) compared with the control group. Pd was significantly greater in the FMS group compared with the control group [46 (29-62) ms vs. 32 (25-37) ms, $p<0.001]$. In the FMS group, there was no significant relationship of the echocardiographic parameters of ACT, Pmax and Pd with age, E/A ratio and deceleration time (DT); while all these five parameters were significantly correlated with left atrial dimension, isovolumetric relaxation time (IVRT), fibromyalgia impact questionnaire (FIQ) and visual analogue scale (VAS). There was a strong correlation between FIQ and VAS and echocardiographic parameters of ACT, Pmax and Pd.

Conclusions: Impaired diastolic functions, an increase in $\mathrm{Pd}$, and prolongation of ACT were observed in FMS. Current disorders are thought to be associated with an increased risk of AF in FMS. The risk of developing AF increases with the severity of FMS and clinical progression.

Keywords: Fibromyalgia syndrome, Atrial fibrillation, Impaired diastolic functions, P wave dispersion, Prolongation of atrial electromechanical interval

\footnotetext{
* Correspondence: drhakkaya@hotmail.com

This article presents new contributions to the development of atrial fibrillation that has not been previously demonstrated in fibromyalgia syndrome. For the first time, it makes new contributions between the severity of fibromyalgia syndrome and the risk of developing atrial fibrillation.

${ }^{1}$ Cardiology Department, Niğde Ömer Halisdemir University Medicine Faculty

Education and Research Hospital, Niğde, Turkey

Full list of author information is available at the end of the article
}

(c) The Author(s). 2020 Open Access This article is distributed under the terms of the Creative Commons Attribution 4.0 International License (http://creativecommons.org/licenses/by/4.0/), which permits unrestricted use, distribution, and reproduction in any medium, provided you give appropriate credit to the original author(s) and the source, provide a link to the Creative Commons license, and indicate if changes were made. The Creative Commons Public Domain Dedication waiver (http://creativecommons.org/publicdomain/zero/1.0/) applies to the data made available in this article, unless otherwise stated. 


\section{Contributions of the paper}

1. There is an increased risk of developing atrial fibrillation in fibromyalgia syndrome.

2. As the severity of fibromyalgia syndrome increases, the risk of developing AF increases.

\section{Introduction}

Fibromyalgia syndrome (FMS) is a non-inflammatory rheumatic syndrome with systemic symptoms such as chronic diffuse pain, fatigue, stiffness, depression, cognitive dysfunction, anxiety and sleep disorders [1].

Although the pathophysiology of FMS is not clear, the dysfunction of the autonomic nervous system is thought to be involved in the etiopathogenesis of the disease. In FMS, both ways of the autonomic nervous system (sympathetic and parasympathetic) are affected at various degrees. Autonomic nervous system dysfunction is responsible for accompanying sleep disorder, anxiety, Raynaud phenomenon and irritable colon syndrome [2].

Atrial fibrillation (AF) is the leading cause of ischemic stroke and is one of the most common arrhythmias [3]. Both parasympathetic and sympathetic nervous system dysfunction are known to trigger potential AF development. Non-invasive tests such as echocardiography and ECG are used to predict the development of AF. The P wave dispersion $(\mathrm{Pd})$, which is especially detected from superficial electrocardiography (ECG), is considered an indirect AF indicator [4]. Prolongation of atrial conduction times (ACT) has been shown to be associated with the risk of AF development and recurrence [5]. There is also a clear association between impaired diastolic functions and AF development [6].

Pd and impaired diastolic functions have been shown in FMS patients [7] but there has been no study on ACT evaluations in FMS. Therefore, the aim of this study was to evaluate the left ventricular diastolic functions, the $\mathrm{ACT}$ and Pd in terms of AF, which may develop in patients with FMS.

\section{Methods}

\section{Study design and participants}

A total of 140 subjects (70 FMS patient group, 70 control group) participated in this case-control study between January 2019 and June 2019. Female patients aged $35-55$ years were included in both groups. The FMS patient group comprised patients diagnosed with fibromyalgia according to the American College of Rheumatology 1990 Criteria for the Classification of Fibromyalgia [8], who were admitted to the Department of Physical Medicine and Rehabilitation. The control group consisted of healthy subjects selected from patients with non-specific symptoms and no cardiovascular or other systemic diseases, who were attending the Department of Physical Medicine and Rehabilitation.

The study was conducted in accordance with the principles of the Declaration of Helsinki. The study protocol was approved by the Niğde Ömer Halisdemir University Clinical Research Ethics Committee. Written informed consent was obtained from each study participant.

Patients were excluded if they had AF, dysrhythmia or taking antiarrhythmic drugs, hypo-hyperthyroidism, ECG abnormalities such as atrioventricular blocks or intrainterventricular conduction defects, severe and moderate valve pathology, atherosclerotic heart disease, cardiomyopathies, pulmonary hypertension, pericardial disease, or a history of pulmonary embolism in both groups. In the FMS group, patients who had previously received treatment of fibromyalgia were excluded.

\section{The fibromyalgia impact questionnaire and visual analogue scale}

The Fibromyalgia Impact Questionnaire (FIQ) was developed to assess the condition, progression, and outcome of fibromyalgia patients. The FIQ consists of 10 items, including physical disability, general well-being, anxiety, dismissal, job success, fatigue, pain, rest, stiffness and depression. The FIQ is based on a specific algorithm, with $\geq 80$ points indicating severe disease [9].

The severity of pain was evaluated with a Visual Analogue Scale (VAS), where 0 points $=$ no pain and 10 points $=$ intolerable pain.

\section{Echocardiographic analysis}

Echocardiographic evaluations were performed by the same cardiologist using a 3.5-MHz transducer (EPIQ 7, Philips Healthcare, Andover, Mass., USA). The clinical diagnosis and status of the patients were not known by the cardiologist. During the echocardiographic evaluation, the patients were monitored and turned to their left sides. All measurements were repeated at least three times and mean values were taken. 2D echocardiographic measurements were performed according to the standards determined by the American Society of Echocardiography [10].

Using the Simpson method, the LV ejection fraction (LVEF) was calculated. The sample volume was placed on the mitral leaflet tips, then pulse wave mitral flow velocities were measured from the apical four chamber view. Mitral early diastolic velocity $(\mathrm{E}, \mathrm{cm} / \mathrm{s})$, late diastolic velocity $(\mathrm{A}, \mathrm{cm} / \mathrm{s}), \mathrm{E} / \mathrm{A}$ ratio, $\mathrm{E}$ deceleration time (DT, ms) and isovolumetric relaxation time (IVRT, $\mathrm{ms}$ ) were calculated from the apical view. On tissue Doppler imaging (TDI) from the lateral and septal aspects of the mitral annulus, measurements were taken of the E' septal and E'lateral early diastolic velocities. 
The E' mean was calculated from the mean of the septal and lateral values.

TDI was used for the evaluation of atrial electromechanical intervals. First, the Nyquist limit was set to 15-20 $\mathrm{cm} / \mathrm{s}$ and the monitor sweeping speed to $50-100 \mathrm{~mm} / \mathrm{s}$ to be able to obtaine the optimum spectral image of myocardial velocities. The PA interval, representing the atrial electromechanical intervals, was defined as the time from the beginning of the P wave on ECG to the beginning of the A wave on TDI. The PA interval was measured from the lateral mitral annulus, the PA lateral, from the septal mitral annulus, the PA septum, and from the right ventricular tricuspid annulus, the PA tricuspid. The left intra-atrial electromechanical delay (EMD) was defined as the difference between lateral and septal PA intervals, the right intra-atrial EMD as the difference between the septal and tricuspid PA intervals, and the inter-atrial EMD as the difference between the lateral and tricuspid PA intervals [11].

\section{Electrocardiographic analysis}

ECG was taken after $10 \mathrm{~min}$ of rest at a speed of $50 \mathrm{~mm} /$ sec and a voltage of $20 \mathrm{~mm} / \mathrm{mV}$. ECG evaluations were performed manually at $\times 10$ magnification by the same cardiologist who was blinded to the clinical details of the patients. $\mathrm{P}$ wave durations were calculated using 12 leads. The longest $\mathrm{p}$ wave duration was Pmax, the shortest $\mathrm{P}$ wave duration was Pmin, and the difference between them was defined as Pd.

\section{Reproducibility}

For evaluation of intraobserver agreement, 20 patients were selected at random, and the measurements were repeated by the same cardiologist under the same conditions. The intra-observer variation coefficients were $6.5 \%$ for PA tricuspid, $5.6 \%$ for PA septum and $5.8 \%$ for PA lateral. The intra-observer coefficient of variation for Pwave variables was $4.8 \%$. These coefficients of variation were not statistically significant.

\section{Statistical analysis}

Statistical analysis was performed using IBM SPSS 20.0 software (IBM Corp., Armonk NY, USA). Categorical variables were summarized as frequency and percentage. Continuous variables were presented as mean \pm standard deviation or median (minimum-maximum) values. The Shapiro-Wilks test was used to evaluate the normality of continuous variables. According to the normality distribution of the continuous variables, differences between the two groups were determined using the Student's $t$ test or the Mann-Whitney $U$ test. Categorical variables were compared with Pearson or the Chi-square test. Pearson correlation coefficient was used to show the relationship between continuous variables. A value of $p<0.05$ was considered statistically significant.

\section{Results}

The laboratory and clinical features of the study groups are given in Table 1. Age, systolic, diastolic and mean blood pressure, hypertension incidence rates, body mass index, serum levels of total cholesterol, fasting blood glucose levels, serum levels of thyroid stimulating hormone, and smoking status were not different between the two groups (Table 1).

There was no significant difference between the FMS and control groups in terms of LVEF, LV end-diastolic dimension (LVEDD), LV end-systolic dimension (LVESD), interventricular septum diameter (IVSD), LV posterior wall diameter (PWD), A wave velocity, E/E' lateral, E/E' septal and E/E' mean.

In the FMS group, the E wave velocity and E/A ratio of the mitral flow were significantly lower, the IVRT was significantly higher and LA diameter were significantly wider, compared to the control group (Table 2).

The septal PA, lateral PA and tricuspid PA values were significantly higher in the FMS group than the control group. The left-sided intra-atrial EMD, right-sided intraatrial EMD and inter-atrial EMD in the FMS group were significantly higher than in the control group (Table 3 ).

In the FMS group the P max was prolonged compared to the control group, but there was no significant change in the P min value. Pd was found to be higher in the FMS group compared to the control group (Table 4).

In the FMS group, there was no significant relationship between left-sided intra-atrial EMD, right-sided intra-atrial EMD, inter-atrial EMD, Pmax and Pd, and age, E/A ratio and DT. All these five parameters were

Table 1 The laboratory and clinical features of the FMS and control groups

\begin{tabular}{llll}
\hline & $\begin{array}{l}\text { FMS group } \\
\mathrm{n}: 70\end{array}$ & $\begin{array}{l}\text { Control group } \\
\mathrm{n}: 70\end{array}$ & $p$ \\
\hline Age (years) & $38.0 \pm 10.8$ & $37.8 \pm 9.9$ & 0.516 \\
SBP (mm Hg) & $123.4 \pm 11.9$ & $121.6 \pm 9.6$ & 0.739 \\
DBP (mm Hg) & $79.8 \pm 9.5$ & $78.6 \pm 8.9$ & 0.577 \\
MBP (mmHg) & $89.43 \pm 8.60$ & $88.86 \pm 9.1$ & 0.651 \\
Hypertension (n (\%)) & $5(7.1)$ & $4(5.7)$ & 0.844 \\
BMI (kg/m2) & $26.6 \pm 5.6$ & $25.2 \pm 4.7$ & 0.284 \\
Total cholesterol (mg/dL) & $179.8 \pm 20.5$ & $178.3 \pm 19.8$ & 0.794 \\
Fasting glucose (mg/dL) & $87.8 \pm 6.7$ & $88.0 \pm 6.6$ & 0.936 \\
TSH (ulU/mL) & $1.99 \pm 0.38$ & $1.87 \pm 0.46$ & 0.528 \\
Serum creatinine, mg/dL & $1.04 \pm 0.12$ & $1.06 \pm 0.14$ & 0.654 \\
Smokers (n (\%)) & $11(15.7)$ & $9(12.8)$ & 0.698 \\
\hline
\end{tabular}

$S B P$ systolic blood pressure, $D B P$ diastolic blood pressure, $M B P$ mean blood pressure, $B M I$ body mass index, TSH Thyroid stimulating hormone, FMS Fibromyalgia syndrome 
Table 2 Conventional echocardiographic and tissue Doppler echocardiographic parameters of the FMS and control groups

\begin{tabular}{llll}
\hline & $\begin{array}{lll}\text { FMS group } \\
\mathrm{n}: 70\end{array}$ & $\begin{array}{l}\text { Control group } \\
\mathrm{n}: 70\end{array}$ & $p$ \\
\hline Heart rate (beat/min) & $82.6 \pm 9.0$ & $81.8 \pm 9.9$ & 0.513 \\
LVEF (\%) & $64.4 \pm 4.8$ & $66.9 \pm 4.4$ & 0.724 \\
LVEDD (cm) & $4.4 \pm 3.0$ & $4.4 \pm 2.7$ & 0.874 \\
LVESD (cm) & $2.9 \pm 0.7$ & $2.8 \pm 0.8$ & 0.816 \\
IVSD (cm) & $0.9 \pm 0.2$ & $0.9 \pm 0.1$ & 0.523 \\
PWD (cm) & $0.9 \pm 0.2$ & $0.9 \pm 0.3$ & 0.687 \\
E (m/s) & $0.71 \pm 0.09$ & $0.76 \pm 0.05$ & 0.035 \\
A (m/s) & $0.63 \pm 0.08$ & $0.61 \pm 0.05$ & 0.366 \\
E/A (Ratio) & $1.15 \pm 0.16$ & $1.26 \pm 0.06$ & 0.001 \\
DT (ms) & $186 \pm 23$ & $179 \pm 21$ & 0.254 \\
IVRT (ms) & $99 \pm 9.4$ & $92 \pm 6.7$ & $<0.001$ \\
E/E' lateral & $6.1 \pm 2.7$ & $6.0 \pm 2.2$ & 0.494 \\
E/E' septal & $7.4 \pm 2.6$ & $7.1 \pm 2.1$ & 0.608 \\
E/E' m & $6.7 \pm 2.3$ & $6.5 \pm 1.8$ & 0.726 \\
LA dimension (cm) & $3.3 \pm 0.4$ & $3.1 \pm 0.3$ & 0.009 \\
\hline
\end{tabular}

LVEF left ventricular ejection fraction, $L V E D D$ left ventricular end-diastolic dimension, LVESD left ventricular end-systolic dimension, IVSD interventricula septum diameter, $P W D$ posterior wall Diameter, $E$ Peak early diastolic velocity, $A$ Peak late diastolic velocity, E/A Ratio of peak early to peak late diastolic velocities, DT Deceleration time, IVRT isovolumetric relaxation time, $E / E^{\prime} m$ Septal and lateral E/E' mean value, LA left atrium, FMS Fibromyalgia syndrome

significantly correlated with LA dimension, IVRT, FIQ and VAS in the FMS group. There was a strong correlation between FIQ and VAS and the echocardiographic parameters of ACT (left-sided EMD, right-sided atrial EMD and inter-atrial EMD), Pmax, Pd (Table 5).

\section{Discussion}

There were four main findings in this study. First, the left and right intra-atrial EMD and inter-atrial EMD were significantly longer in patients with FMS than in the control group. Second, the Pmax and Pd values were higher in patients with FMS than in the control group. The $\mathrm{E}$ wave duration and E/A ratio were significantly

Table 3 The atrial conduction times of the FMS and control groups

\begin{tabular}{llll}
\hline & FMS group & $\begin{array}{l}\text { Control group } \\
\text { n:70 }\end{array}$ & $p$ \\
\hline Septal PA (ms) & $63(56-84)$ & $51(44-57)$ & $<0.001$ \\
Lateral PA (ms) & $84(66-110)$ & $54(51-62)$ & $<0.001$ \\
Tricuspid PA (ms) & $45(39-54)$ & $40(38-56)$ & $<0.001$ \\
Left-sided intra-atrial EMD (ms) & $13.9 \pm 5.9$ & $8.1 \pm 1.8$ & $<0.001$ \\
Right-sided intra-atrial EMD (ms) & $21.9 \pm 8.2$ & $10.4 \pm 3.5$ & $<0.001$ \\
Inter-atrial EMD (ms) & $40(25-64)$ & $23(14-27)$ & $<0.001$ \\
\hline
\end{tabular}

EMD electromechanical delay, FMS Fibromyalgia syndrome
Table 4 Electrocardiographic parameters of FMS and control groups

\begin{tabular}{llll}
\hline & $\begin{array}{l}\text { FMS group } \\
\mathrm{n}: 70\end{array}$ & $\begin{array}{l}\text { Control group } \\
\mathrm{n}: 70\end{array}$ & $p$ \\
\hline Heart rate (beats/min) & $82.6 \pm 9.0$ & $81.8 \pm 9.9$ & 0.513 \\
Pmax (ms) & $106 \pm 9.4$ & $96 \pm 5.6$ & $<0.001$ \\
Pmin (ms) & $68 \pm 3.9$ & $69 \pm 4.1$ & 0.394 \\
Pd (ms) & $46(29-62)$ & $32(25-37)$ & $<0.001$ \\
\hline
\end{tabular}

$P d$ P-wave dispersion, Pmax maximum P-wave duration, Pmin minimum Pwave duration, FMS Fibromyalgia syndrome

decreased and IVRT was significantly increased in the FMS patient group compared to the control group. These parameters are indicative of moderate diastolic dysfunction. The diameter of the left atrium was larger in the FMS patient group than in the control group. In addition, the echocardiographic parameters of ACT were significantly correlated with echocardiographic indices of diastolic functions, Pmax and Pd in patients with FMS. Finally, the FIQ and VAS, which are indicative of disease severity, were correlated with Pmax, Pd and delay of $\mathrm{ACT}$ in the FMS patient group.

Neurohormonal factors and autonomic dysfunction have been implicated in the etiopathogenesis of FMS [12]. Autonomic dysfunction manifests as sympathetic hyperactivity and decreased parasympathetic activity [13]. It is known that impaired heart innervation may cause ventricular dysfunction, including hyperactivity of sympathetic fibers, peripheral vasoconstriction, inotropic increase in the heart and indirectly, cause harmful reactions in myocytes [14]. The autonomic nervous system disrupts the sinoatrial node and the atrioventricular node, both of which can be affected by autonomic stimuli. In the absence of any other reasons, autonomic dysfunction has been accepted as the cause of arrhythmias [12].

Previous clinical studies have shown increased arrhythmias [12], increased carotid intima media thickness [15], non-dipper circadian blood pressure change [16], increased coronary artery disease [17], arterial stiffness [18] and diastolic dysfunction [19] in FMS patient groups. In these clinical studies, autonomic dysfunction and neurohumoral factors have been implicated in the etiopathogenesis of these clinical conditions with FMS.

$\mathrm{AF}$, which is the most common cause of ischaemic stroke, can manifest with palpitations, heart failure, increased hospitalization, and reduced exercise capacity. Inter-atrial and intra EMD have been shown to be associated with new-onset and relapse of AF in patients with or without heart disease $[5,20]$. Pd is considered a specific and sensitive marker of AF [21] and has been reported to be an indicator of paroxysmal AF [22]. Left ventricular diastolic dysfunction also increases the risk of developing AF [6]. 
Table 5 Correlations between echocardiographic parameters of atrial conduction times, electrocardiographic parameters, clinical and laboratory characteristics of patients with FMS

\begin{tabular}{|c|c|c|c|c|c|c|c|c|c|c|}
\hline \multirow[t]{2}{*}{ Parameters } & \multicolumn{2}{|c|}{$\begin{array}{l}\text { Left-sided } \\
\text { EMD (ms) }\end{array}$} & \multicolumn{2}{|c|}{$\begin{array}{l}\text { Right-sided } \\
\text { EMD (ms) }\end{array}$} & \multicolumn{2}{|c|}{$\begin{array}{l}\text { Interatrial } \\
\text { EMD (ms) }\end{array}$} & \multicolumn{2}{|c|}{ Pmax (ms) } & \multicolumn{2}{|c|}{$\mathrm{Pd}$ (ms) } \\
\hline & r & $p$ & $r$ & $p$ & r & $p$ & $r$ & $p$ & r & $p$ \\
\hline Age (years) & 0.036 & 0.852 & 0.176 & 0.284 & 0.156 & 0.360 & 0.144 & 0.436 & 0.098 & 0.812 \\
\hline E/A ratio & 0.064 & 0.695 & -.130 & 0.424 & 0.061 & 0.709 & 0.366 & 0.066 & 0.384 & 0.060 \\
\hline DT (ms) & 0.368 & 0.019 & 0.024 & 0.885 & 0.226 & 0.162 & 0.342 & 0.096 & 0.364 & 0.102 \\
\hline IVRT (ms) & 0.366 & 0.020 & 0.394 & 0.012 & 0.501 & 0.001 & 0.798 & $<0.001$ & 0.786 & $<0.001$ \\
\hline LA dimension $(\mathrm{cm})$ & 0.675 & $<0.001$ & 0.804 & $<0.001$ & 0.856 & $<0.001$ & 0.765 & $<0.001$ & 0.698 & $<0.001$ \\
\hline Pmax (ms) & 0.668 & $<0.001$ & 0.690 & $<0.001$ & 0.892 & $<0.001$ & 1 & & 0.898 & $<0.001$ \\
\hline $\mathrm{Pd}(\mathrm{ms})$ & 0.660 & $<0.001$ & 0.682 & $<0.001$ & 0.886 & $<0.001$ & 0.898 & $<0.001$ & 1 & \\
\hline $\mathrm{FIQ}$ & 0.668 & $<0.001$ & 0.688 & $<0.001$ & 0.888 & $<0.001$ & 0.868 & $<0.001$ & 0.866 & $<0.001$ \\
\hline VAS & 0.904 & $<0.001$ & 0.796 & $<0.001$ & 0.764 & $<0.001$ & 0.820 & $<0.001$ & 0.754 & $<0.001$ \\
\hline
\end{tabular}

EMD electromechanical delay, E/A Ratio of peak early to peak late diastolic velocities, DT deceleration time, IVRT isovolumetric deceleration time, $L A$ left atrium, Pmax maximum P-wave duration, Pd P-wave dispersion, Pmax minimum P-wave duration, FIQ Fibromyalgia Impact Questionnaire, VAS Visual Analogue Scale

In previous studies, changes in $\mathrm{p}$-wave duration and the presence of diastolic dysfunction have been shown to be higher in FMS patients [19, 23]. To the best of our knowledge, there has beeen no previous study on ACT in FMS patients. In the current study, changes in $\mathrm{p}$ wave durations and prolongation of ACT were observed, and these findings support an increased risk of developing AF in the FMS patient group. The increased diastolic dysfunction also supports this condition.

Catecholamine and adrenergic discharge disorders and permanent activation of catecholamine-induced calcium channels are thought to be possible mechanisms for increased arterial stiffness and diastolic dysfunction in FMS patients $[24,25]$. The prolongation of $\mathrm{p}$ wave durations and prolongation of ACT may have been the result of all these pathologies and left atrial enlargement.

FIQ and VAS values reflect the severity of the disease. In previous studies, a correlation has been shown between FIQ and VAS values and vascular stiffness [18]. On this basis, it is possible to observe the correlation between FIQ and VAS values and Pmax, Pd and ACT in our study. It has thus been shown for the first time in literature that the risk of AF development may be increased with increased disease severity.

\section{Study limitations}

The main limiting factor of this study was the lack of evaluation of autonomic nervous system dysfunction in both groups. The study was a cross-sectional study and so may not reflect all FMS patients and long-term follow-up of these patient groups was not performed. ECG holter monitoring that can detect occult arrhythmias and heart rate variability indicating autonomic nervous system dysfunction was not used in the follow-up of patients, and there was no evaluation of serum catecholamine levels. The inclusion of only female subjects can be considered another limitation and the relatively low number of patients.

\section{Conclusions}

In conclusion, the results of this study demonstrated that the ACT were longer and the Pd value was higher in FMS patients than in the control group. Impaired diastolic dysfunctions were also determined in FMS patients, and it was seen that the risk of AF development may be increased by the severity of the disease. Although no previous long-term follow-up studies have shown an increased risk of AF, all these abnormalities may increase the risk of cardiovascular and cerebrovascular events in patients with FMS.

\section{Acknowledgements}

None.

\section{Authors' contributions}

HA: study design, acquisition of data, writing and drafting the manuscript, and final approval; guarantor of the study, correspondence author. EEG: study design, acquisition of data, writing and drafting the manuscript, and final approval, EK: study design, statistical analyses, writing and drafting the manuscript, and final approval. BÖ: study design, acquisition of data, writing and drafting the manuscript, and final approval.

\section{Funding}

No funding was received.

\section{Availability of data and materials \\ The data that support the findings of this study are available from the corresponding author, upon reasonable request.}

\section{Ethics approval and consent to participate}

We declare that this study was approved by the Niğde Ömer Halisdemir University Clinical Research Ethics Committee in the application no. 2019/01 and was therefore conducted in accordance with the ethical standards set forth in the 1964 Declaration of Helsinki and its subsequent amendments. All patients gave their informed consent prior to their inclusion in the study. Details that may reveal the identity of the subjects under study have been omitted. 


\section{Consent for publication}

All authors are aware of the full content of the manuscript and provided consent for the submission to Advances in Rheumatology.

\section{Competing interests}

The authors declare that they have no competing interests.

\section{Author details}

${ }^{1}$ Cardiology Department, Niğde Ömer Halisdemir University Medicine Faculty Education and Research Hospital, Niğde, Turkey. ${ }^{2}$ Physical Medicine and Rehabilitation Department, Niğde Ömer Halisdemir University Medicine Faculty Education and Research Hospital, Niğde, Turkey.

Received: 23 October 2019 Accepted: 19 January 2020

Published online: 30 January 2020

\section{References}

1. Güler H, Yıldızgören MT, Üstün N, Paksoy H, Turhanoglu AD. Isokinetic assessment of the wrist muscles in females with fibromyalgia. Arch Rheumatol. 2016;31:215-20.

2. Kulshreshtha P, Gupta R, Yadav RK, Bijlani RL, Deepak KK. A comprehensive study of autonomic dysfunction in the fibromyalgia patients. Clin auton res. 2012:22:117-22.

3. Go AS, Hylek EM, Phillips KA, Chang Y, Henault LE, Selby JV, et al. Prevalence of diagnosed atrial fibrillation in adults: national implications for rhythm management and stroke prevention: the anticoagulation and risk factors in atrial fibrillation (ATRIA) study. JAMA. 2001;285:2370-5.

4. Podrid PJ, Kowney PR.(2001) Cardaic arrhythmia, mechanisms, diagnosis \& management. 2nd ed. Philadelphia: Lippincott Williams \& Williams; p. 111-651.

5. Pytkowski M, Jankowska A, Maciag A, Kowalik I, Sterlinski M, Szwed H, et al. Paroxysmal atrial fibrillation is associated with increased intraatrial conduction delay. Europace. 2008;10:1415-20.

6. Delgado V, Bax J. Diastolic dysfunction and atrial fibrillation. Heart. 2015; 101(16):1263-4

7. Sarifakioglu B, Guzelant AY, Alpsoy S, Topcu B, Unsal C, Sahin N. Is there a new finding added to the fibromyalgia syndrome? North Clin Istanbul. 2014;1(1):6-12.

8. Wolfe F, Smythe HA, Yunus MB, Bennett RM, Bombardier C, Goldenberg DL, et al. The American College of Rheumatology 1990 criteria for the classification of fibromyalgia. Report of the multicenter criteria committee. Arthritis Rheum. 1990;33:160-72.

9. Bennett $\mathrm{R}$. The fibromyalgia impact questionnaire $(\mathrm{FIQ})$ : a review of its development, current version, operating characteristics and uses. Clin Exp Rheumatol. 2005:23:154-62.

10. Gottdiener JS, Bednarz J, Devereux R, Gardin J, Klein A, Manning WJ, et al. American Society of Echocardiography recommendations for use of echocardiography in clinical trials. J Am Soc Echocardiogr. 2004;17:1086119.

11. Cui QQ, Zhang W, Wang H, Sun X, Wang R, Yang HY, et al. Assessment of atrial electromechanical coupling and influential factors in nonrheumatic paroxysmal atrial fibrillation. Clin Cardiol. 2008;31:74-8.

12. Dogru MT, Aydin G, Tosun A, Keleş I, Güneri M, Arslan A, et al. Correlations between autonomic dysfunction and circadian changes and arrhythmia prevalence in women with fibromyalgia syndrome. Anadolu Kardiyol Derg. 2009;9:110-7.

13. Grassi G, Seravalle G, Cattaneo BM, Lanfranchi A, Vailati S, Giannattasio C, et al. Sympathetic activation and loss of reflex sympathetic control in mild congestive heart failure. Circulation. 1995;92:3206-11.

14. Deswal A. Diastolic dysfunction and diastolic heart failure: mechanisms and epidemiology. Curr Cardiol Rep. 2005;7:178-83.

15. Bölük H, Öztürk GT, Cömert D, Ersöz M. Increased carotid intima-media thickness in female patients with fibromyalgia: a preliminary study. Arch Rheumatol. 2015;30:307-10.

16. Inal S, Inal EE, Okyay GU, Öztürk GT, Öneç K, Güz G. Fibromyalgia and nondipper circadian blood pressure variability. J Clin Rheumatol. 2014; 20:422-6.

17. Tsai PS, Fan YC, Huang CJ. Fibromyalgia is associated with coronary heart disease: a population-based cohort study. Reg Anesth Pain Med. 2015;40: $37-42$.
18. Gunturk EE, Ekiz T, Akkaya H. Evaluation of carotid-femoral pulse wave velocity, aortic stiffness index, and aortic distensibility in patients with fibromyalgia Clin Rheumatol. 2019;38(6):1731-5.

19. Sayilir $\mathrm{S}$, Ergün $\mathrm{G}$, Ekiz T. Evaluation of ventricular diastolic function in patients with fibromyalgia syndrome. Arch Rheumatol. 2017;33(2):150-3.

20. De Vos CB, Weijs B, Crijns HJ, Cheriex EC, Palmans A, Habets J, et al. Atrial tissue Doppler imaging for prediction of new-onset atrial fibrillation. Heart. 2009;95:835-40.

21. Dilaveris PE, Gialafos JE. P-wave dispersion: a novel predictor of paroxysmal atrial fibrillation. Ann Noninvasive Electrocardiol. 2001;6:159-65.

22. Aytemir K, Ozer N, Atalar E, Sade E, Aksöyek S, Ovünç K, et al. P wave dispersion on 12-lead electrocardiography in patients with paroxysmal atrial fibrillation. Pacing Clin Electrophysiol. 2000;23:1109-12.

23. Yolbaş S, Yıldııım A, Düzenci D, Karakaya B, Dağlı MN, Koca SS. QT dispersion and $\mathrm{P}$ wave dispersion in patients with fibromyalgia. Eur 」 Rheumatol. 2016;3(4):165-8.

24. Lee JH, Cho Kl, Kim SM, Lee HG, Kim TI. Arterial stiffness in female patients with fibromyalgia and its relationship to chronic emotional and physical stress. Korean Circ J. 2011;41:596-602.

25. Light KC, Bragdon EE, Grewen KM, Brownley KA, Girdler SS, Maixner W. Adrenergic dysregulation and pain with and without acute beta-blockade in women with fibromyalgia and temporomandibular disorder. J Pain. 2009;10:542-52

\section{Publisher's Note}

Springer Nature remains neutral with regard to jurisdictional claims in published maps and institutional affiliations.
Ready to submit your research? Choose BMC and benefit from:

- fast, convenient online submission

- thorough peer review by experienced researchers in your field

- rapid publication on acceptance

- support for research data, including large and complex data types

- gold Open Access which fosters wider collaboration and increased citations

- maximum visibility for your research: over $100 \mathrm{M}$ website views per year

At BMC, research is always in progress.

Learn more biomedcentral.com/submission 